Article

\title{
Subclasses of Multivalent Analytic Functions Associated with a $q$-Difference Operator
}

\author{
Ekram Elsayed Ali ${ }^{1,2,+}$ (D) and Teodor Bulboacă ${ }^{3, *,+}$ (iD \\ 1 Department of Mathematics, Faculty of Science, University of Ha'il, $\mathrm{Ha}^{\prime}$ il 81451, Saudi Arabia; \\ ekram_008eg@yahoo.com \\ 2 Department of Mathematics and Computer Science, Faculty of Science, Port Said University, \\ Port Said 42521, Egypt \\ 3 Faculty of Mathematics and Computer Science, Babeş-Bolyai University, 400084 Cluj-Napoca, Romania \\ * Correspondence: bulboaca@math.ubbcluj.ro; Tel.: +40-729087153 \\ + These authors contributed equally to this work.
}

Received: 14 November 2020; Accepted: 5 December 2020; Published: 8 December 2020

check for updates

\begin{abstract}
In this article we introduced and studied some inclusion properties for new subclasses of multivalent analytic functions defined by using the $q$-derivative operator. With the aid of the Jackson $q$-derivative we defined two new operators that generalize many other previously studied operators, and help us to define two new subclasses of functions with several interesting properties studied in this paper. The methods used for the proof of our results are special tools of the differential subordination theory of one-variable functions.
\end{abstract}

Keywords: analytic function; Hadamard (convolution) product; $q$-derivative operator; multivalent functions

MSC: 30C45

\section{Introduction and Preliminaries}

Let $\mathbb{A}(p)$ denote the class of functions of the form

$$
f(z)=z^{p}+\sum_{k=1}^{\infty} a_{k+p} z^{k+p}, z \in \mathbb{D}, p \in \mathbb{N}:=\{1,2, \ldots\}
$$

that are analytic multivalent in the open unit disc $\mathbb{D}:=\{z \in \mathbb{C}:|z|<1\}$. We denote by $f * h$ the Hadamard (or convolution) product of the functions $f$ and $h$ analytic in $\mathbb{D}$, that is, if $f$ is given by (1) and

$$
h(z)=z^{p}+\sum_{k=1}^{\infty} b_{k+p} z^{k+p}, z \in \mathbb{D},
$$

then

$$
(f * h)(z):=z^{p}+\sum_{k=1}^{\infty} a_{k+p} b_{k+p} z^{k+p}, z \in \mathbb{D} .
$$

For $0<q<1$, in $[1,2]$ Jackson defined the $q$-derivative operator $D_{q}$ of a function $f$ by

$$
D_{q} f(z):= \begin{cases}\frac{f(z)-f(q z)}{(1-q) z}, & \text { if } z \neq 0, \\ f^{\prime}(0) . & \text { if } z=0,\end{cases}
$$


From (2) it follows that if $f \in \mathbb{A}(p)$ has the form (1), then

$$
D_{q} f(z)=D_{q}\left(z^{p}+\sum_{k=1}^{\infty} a_{k+p} z^{k+p}\right)=[p]_{q} z^{p-1}+\sum_{k=1}^{\infty}[k+p]_{q} a_{k+p} z^{k+p-1}, z \in \mathbb{D},
$$

where $[k]_{q}:=\frac{1-q^{k}}{1-q}$, and thus $\lim _{q \rightarrow 1}[k]_{q}=k$.

Using the above Jackson $q$-derivative we will define the operator $\partial_{p, q}^{n}: \mathbb{A}(p) \rightarrow \mathbb{A}(p), n \in \mathbb{N}_{0}:=$ $\mathbb{N} \cup\{0\}$, by

$$
\partial_{p, q}^{0} f(z):=f(z), \quad \partial_{p, q}^{n} f(z):=z D_{q}\left(\partial_{p, q}^{n-1} f(z)\right), n \in \mathbb{N} .
$$

Therefore, if $f \in \mathbb{A}(p)$ has the form (1) it follows that

$$
\partial_{p, q}^{n} f(z)=\left(f * G_{p, q}^{n}\right)(z), z \in \mathbb{D}, p \in \mathbb{N}_{0},
$$

where

$$
G_{p, q}^{n}(z):=z^{p}+\sum_{k=1}^{\infty}\left([k+p]_{q}\right)^{n} z^{k+p}, z \in \mathbb{D}, p \in \mathbb{N}, n \in \mathbb{N}_{0}
$$

Moreover,

$$
\partial_{p, q}^{n} f(z)=z^{p}+\sum_{k=1}^{\infty}\left([k+p]_{q}\right)^{n} a_{k+p} z^{k+p}, z \in \mathbb{D},
$$

and

$$
\lim _{q \rightarrow 1^{-}} \partial_{p, q}^{n} f(z)=z^{p}+\sum_{k=1}^{\infty}(k+p)^{n} a_{k+p} z^{k+p}, z \in \mathbb{D} .
$$

For $\eta \geq 0$, with the aid of the operator $\partial_{p, q}^{n}$ we will define the new $q$-differential operator $\mathfrak{S}_{\eta, p, q}^{n, m}$ : $\mathbb{A}(p) \rightarrow \mathbb{A}(p)$ by

$$
\begin{aligned}
& \mathfrak{S}_{\eta, p, q}^{n, 0} f(z):=\partial_{p, q}^{n} f(z), \\
& \mathfrak{S}_{\eta, p, q}^{n, m} f(z):=(1-\eta) \mathfrak{S}_{\eta, p, q}^{n, m-1} f(z)+\eta \frac{z}{p}\left(\mathfrak{S}_{\eta, p, q}^{n, m-1} f(z)\right)^{\prime}, m \in \mathbb{N} .
\end{aligned}
$$

From the above definition it follows easily that if $f \in \mathcal{A}(p)$ is of the form (1), then

$$
\mathfrak{S}_{\eta, p, q}^{n, m} f(z)=z^{p}+\sum_{k=1}^{\infty}\left([k+p]_{q}\right)^{n}\left(\frac{p+\eta k}{p}\right)^{m} a_{k+p} z^{k+p}, z \in \mathbb{D}, m \in \mathbb{N}_{0} .
$$

Remark 1. 1. For $p=1$ the $q$-differential operator $\mathfrak{S}_{\eta, p, q}^{n, m}$ reduces to the $q$-differential operator defined by Frasin and Murugusundaramoorthy [3].

2. If $q \rightarrow 1^{-}$we get the differential operator

$$
\mathcal{S}_{\eta, p}^{n, m} f(z):=z^{p}+\sum_{k=1}^{\infty}(k+p)^{n}\left(\frac{p+\eta k}{p}\right)^{m} a_{k+p} z^{k+p}, z \in \mathbb{D}, m, n \in \mathbb{N}_{0}
$$

3. By specializing the parameters $\eta, m, n$ and $p$ we obtain the following operators studied by various authors:

(i) $\mathfrak{S}_{\eta, p}^{0, m}=: D_{\eta, p}^{m}$ (see Aouf et al. [4]);

(ii) $\mathfrak{S}_{1, p}^{0, m}=: D_{p}^{m}$ (see [5-7]);

(iii) $\mathfrak{S}_{1,1}^{n, 0}=: D^{n}, m \in N_{0}$ (see Sălăgean [8]);

(iv) $\mathfrak{S}_{\eta, 1}^{0, m}=: D_{\eta}^{m}$ (see Al-Aboudi [9]).

4. Recently, many researches connected with fractional-order integral and derivative operators have been published (for example, see [10-13]). 
Definition 1. 1. We denote by $M_{\eta, p, q}^{n, m}(\alpha ; \beta)$ the subclass of $\mathbb{A}(p)$ consisting of functions $f \in \mathbb{A}(p)$ that satisfy the inequality

$$
\operatorname{Re}\left\{(1-\alpha) \frac{z\left(\mathfrak{S}_{\eta, p, q}^{n, m} f(z)\right)^{\prime}}{\mathfrak{S}_{\eta, p, q}^{n, m} f(z)}+\alpha\left(1+\frac{z\left(\mathfrak{S}_{\eta, p, q}^{n, m} f(z)\right)^{\prime \prime}}{\left(\mathfrak{S}_{\eta, p, q}^{n, m} f(z)\right)^{\prime}}\right)\right\}>\beta, \in \mathbb{D}
$$

where $\alpha \in \mathbb{R}$ and $\beta<1$.

2. Let $A_{\eta, p, q}^{n, m}(\lambda, \vartheta ; \gamma)$ be the subclass of $\mathbb{A}(p)$ consisting of functions $f \in \mathbb{A}(p)$ that satisfy the conditions

$$
\frac{\mathfrak{S}_{\eta, p, q}^{n, m} f(z)\left(\mathfrak{S}_{\eta, p, q}^{n, m} f(z)\right)^{\prime}}{z^{2 p-1}} \neq 0, z \in \mathbb{D}
$$

and

$$
\operatorname{Re}\left\{\left[\frac{\left(\mathfrak{S}_{\eta, p, q}^{n, m} f(z)\right)^{\prime}}{p z^{p-1}}\right]^{\lambda}\left[\frac{\mathfrak{S}_{\eta, p, q}^{n, m} f(z)}{z^{p}}\right]^{\vartheta}\right\}>\gamma, z \in \mathbb{D}
$$

where $\lambda, \vartheta \in \mathbb{R}$ and $\gamma<1$.

We note that the values of the above-mentioned complex powers are taken as their principal values here and throughout this paper.

Remark 2. The families $M_{\eta, p, q}^{n, m}(\alpha ; \beta)$ and $A_{\eta, p, q}^{n, m}(\lambda, \vartheta ; \gamma)$ contain many well-known, as well as many classes of analytic multivalent functions.

(i) For $\lambda=1, \vartheta=-1, n=0, m=0$, and $q \rightarrow 1^{-}$we obtain the family of $p$-valent starlike functions of order $\gamma, 0 \leq \gamma<1$, denoted by $\mathcal{S}_{p}(\gamma)$;

(ii) For $\alpha=1, \beta=\gamma, n=0, m=0, q \rightarrow 1^{-}$we obtain the family of $p$-valent convex functions of order $\gamma, 0 \leq \gamma<1$, denoted by $K_{p}(\gamma)$. We mention that the classes $\mathcal{S}_{p}(\gamma)$ and $K_{p}(\gamma)$ were introduced by Patil and Thakare [14] and Owa [15].

Our analysis deals on certain disparities a differential operator $\Omega_{\eta, p, q}^{n, m}(\lambda, \vartheta): \mathbb{A}(p) \rightarrow \mathbb{A}(p)$ defined by

$$
\Omega_{\eta, p, q}^{n, m}(\lambda, \vartheta) f(z):=\vartheta \frac{z\left(\mathfrak{S}_{\eta, p, q}^{n, m} f(z)\right)^{\prime}}{\mathfrak{S}_{\eta, p, q}^{n, m} f(z)}+\lambda\left(1+\frac{z\left(\mathfrak{S}_{\eta, p, q}^{n, m} f(z)\right)^{\prime \prime}}{\left(\mathfrak{S}_{\eta, p, q}^{n, m} f(z)\right)^{\prime}}\right)
$$

with $\lambda, \vartheta \in \mathbb{R}$, since $f \in M_{\eta, p, q}^{n, m}(\alpha ; \beta)$ if and only if $\operatorname{Re} \Omega_{\eta, p, q}^{n, m}(\alpha, 1-\alpha) f(z)>\beta, z \in \mathbb{D}$.

To prove our main results that generalize the recent results obtained by Irmak et al. [16] we need the following lemmas. More general forms of these lemmas that are very useful in the theory of differential subordinations are due to S. S. Miller and P. T. Mocanu [17] (Theorem 2.3h. and Theorem 2.3i.).

For $m \in \mathbb{N}$ and $a \in \mathbb{C}$ we denote by $\mathcal{H}[a, m]$ the class of all functions $p$ that are analytic in the unit disc $\mathbb{D}$ with the power series expansion of the form

$$
\mathrm{p}(z)=a+a_{m} z^{m}+a_{m+1} z^{m+1}+\ldots, z \in \mathbb{D} .
$$

Lemma 1 ([17]). Let $\Omega \subset \mathbb{C}$ and suppose the function $\psi: \mathbb{C}^{2} \times \mathbb{D} \rightarrow \mathbb{C}$ satisfies $\psi\left(N e^{i \theta}, K e^{i \theta} ; z\right) \notin \Omega$ for all the values of $K$ such that $K \geq m N, \theta \in \mathbb{R}$ and $z \in \mathbb{D}$. If $\mathrm{p} \in \mathcal{H}[0, m]$ and $\psi\left(\mathrm{p}(z), z \mathrm{p}^{\prime}(z) ; z\right) \in \Omega$ for all $z \in \mathbb{D}$, then $|\mathrm{p}(z)|<N, z \in \mathbb{D}$.

Lemma 2 ([17]). Let $\Omega \subset \mathbb{C}$ and suppose the function $\psi: \mathbb{C}^{2} \times \mathbb{D} \rightarrow \mathbb{C}$ satisfies $\psi(i x, y ; z) \notin \Omega$ for all $x, y \in \mathbb{R}, y \leq-m\left(1+x^{2}\right) / 2$ and $z \in \mathbb{D}$. If $\mathrm{p} \in \mathcal{H}[1, m]$ and $\psi\left(\mathrm{p}(z), z \mathrm{p}^{\prime}(z) ; z\right) \in \Omega$ for all $z \in \mathbb{D}$, then $\operatorname{Rep}(z)>0, z \in \mathbb{D}$. 


\section{Main Results}

Now we will prove each of our main results given by the following theorems.

Theorem 1. Let the function $f \in \mathbb{A}(p)$ such that (3) holds, and let $\lambda, \vartheta \in \mathbb{R}$. If

$$
\operatorname{Re} \Omega_{\eta, p, q}^{n, m}(\lambda, \vartheta) f(z)<p(\lambda+\vartheta)+\frac{N}{N+1}, z \in \mathbb{D},
$$

with $N \geq 1$, then

$$
\left|\left[\frac{\left(\mathfrak{S}_{\eta, p, q}^{n, m} f(z)\right)^{\prime}}{p z^{p-1}}\right]^{\lambda}\left[\frac{\mathfrak{S}_{\eta, p, q}^{n, m} f(z)}{z^{p}}\right]^{\vartheta}-1\right|<N, z \in \mathbb{D},
$$

and furthermore $f \in A_{\eta, p, q}^{n, m}(\lambda, \vartheta ; 1-N)$.

Proof. Define the function $p$ by

$$
\mathrm{p}(z)=\left[\frac{\left(\mathfrak{S}_{\eta, p, q}^{n, m} f(z)\right)^{\prime}}{p z^{p-1}}\right]^{\lambda}\left[\frac{\mathfrak{S}_{\eta, p, q}^{n, m} f(z)}{z^{p}}\right]^{\vartheta}-1, z \in \mathbb{D} .
$$

From the assumption (3) it follows that the function $\mathrm{p}$ function is analytic in $\mathbb{D}$, and $\mathrm{p}(0)=0$, that is $\mathrm{p} \in \mathcal{H}[0,1]$. A simple computation shows that

$$
\Omega_{\eta, p, q}^{n, m}(\lambda, \vartheta) f(z)=p(\lambda+\vartheta)+\frac{z \mathrm{p}^{\prime}(z)}{\mathrm{p}(z)+1}, z \in \mathbb{D} .
$$

Now, letting

$$
\begin{aligned}
& \psi(r, s ; z):=p(\lambda+\vartheta)+\frac{s}{r+1}, s \in \mathbb{C}, r \in \mathbb{C} \backslash\{-1\} \\
& \Omega:=\left\{w \in \mathbb{C}: \operatorname{Re} w<p(\lambda+\vartheta)+\frac{N}{N+1}\right\}
\end{aligned}
$$

the assumption (4) is equivalent to $\psi\left(\mathrm{p}(z), z \mathrm{p}^{\prime}(z) ; z\right)=\Omega_{\eta, p, q}^{n, m}(\lambda, \vartheta) f(z) \in \Omega$ all $z \in \mathbb{D}$.

For any $\theta \in \mathbb{R}, K \geq N$ and $z \in \mathbb{D}$, since $N \geq 1$ we obtain that

$$
\operatorname{Re} \psi\left(N e^{i \theta}, K e^{i \theta} ; z\right)=p(\lambda+\vartheta)+K \operatorname{Re} \frac{1}{N+e^{-i \theta}} \geq p(\lambda+\vartheta)+\frac{N}{N+1}
$$

which shows that $\psi\left(N e^{i \theta}, K e^{i \theta} ; z\right) \notin \Omega$ whenever $\theta \in \mathbb{R}, K \geq N$ and $z \in \mathbb{D}$. Therefore, according to Lemma 1 we obtain $|\mathrm{p}(z)|<N$ for all $z \in \mathbb{D}$, that is (5) holds.

For the special case $N=\gamma+1$ the above theorem reduces to the next result, which represents a sufficient condition for a function $f \in \mathbb{A}(p)$ to be in the class $A_{\eta, p, q}^{n, m}(\lambda, \vartheta ;-\gamma)$ :

Corollary 1. Let the function $f \in \mathbb{A}(p)$ such that (3) holds, and let $\lambda, \vartheta \in \mathbb{R}$. If

$$
\operatorname{Re} \Omega_{\eta, p, q}^{n, m}(\lambda, \vartheta) f(z)<p(\lambda+\vartheta)+\frac{\gamma+1}{\gamma+2}, z \in \mathbb{D},
$$

with $\gamma \geq 0$, then

$$
\left|\left[\frac{\left(\mathfrak{S}_{\eta, p, q}^{n, m} f(z)\right)^{\prime}}{p z^{p-1}}\right]^{\lambda}\left[\frac{\mathfrak{S}_{\eta, p, q}^{n, m} f(z)}{z^{p}}\right]^{\vartheta}-1\right|<\gamma+1, z \in \mathbb{D}
$$

therefore $f \in A_{\eta, p, q}^{n, m}(\lambda, \vartheta ;-\gamma)$. 
If we set $\lambda=\alpha$ and $\vartheta=1-\alpha$ in the above corollary we obtain the following result:

Corollary 2. Let the function $f \in \mathbb{A}(p)$ such that (3) holds, and let $\alpha \in \mathbb{R}$. If

$$
\operatorname{Re} \Omega_{\eta, p, q}^{n, m}(\alpha, 1-\alpha) f(z)<p+\frac{\gamma+1}{\gamma+2}, z \in \mathbb{D},
$$

with $\gamma \geq 0$, then

$$
\left|\left[\frac{\left(\mathfrak{S}_{\eta, m, q}^{n, m} f(z)\right)^{\prime}}{p z^{p-1}}\right]^{\alpha}\left[\frac{\mathfrak{S}_{\eta, p, q}^{n, m} f(z)}{z^{p}}\right]^{1-\alpha}-1\right|<\gamma+1, z \in \mathbb{D},
$$

therefore $f \in A_{\eta, p, q}^{n, m}(\alpha, 1-\alpha ;-\gamma)$.

For $\alpha=1$ and $\alpha=0$ the above corollary reduces to the following examples, respectively:

Example 1. Let $\gamma \geq 0$ and $f \in \mathbb{A}(p)$.

(i) If

$$
\operatorname{Re}\left(1+\frac{z\left(\mathfrak{S}_{\eta, p, q}^{n, m} f(z)\right)^{\prime \prime}}{\left(\mathfrak{S}_{\eta, p, q}^{n, m} f(z)\right)^{\prime}}\right)<p+\frac{\gamma+1}{\gamma+2}, z \in \mathbb{D},
$$

then

$$
\left|\frac{\left(\mathfrak{S}_{\eta, m, q}^{n, m} f(z)\right)^{\prime}}{p z^{p-1}}-1\right|<\gamma+1, z \in \mathbb{D},
$$

hence $f \in A_{\eta, p, q}^{n, m}(1,0 ;-\gamma)$.

(ii) If

$$
\operatorname{Re} \frac{z\left(\mathfrak{S}_{\eta, p, q}^{n, m} f(z)\right)^{\prime}}{\mathfrak{S}_{\eta, m, q}^{n, m} f(z)}<p+\frac{\gamma+1}{\gamma+2}, z \in \mathbb{D},
$$

then

$$
\left|\frac{\mathfrak{S}_{\eta, p, q}^{n, m} f(z)}{z^{p}}-1\right|<\gamma+1, z \in \mathbb{D},
$$

hence $f \in A_{\eta, p, q}^{n, m}(0,1 ;-\gamma)$.

Theorem 2. Let the function $f \in \mathbb{A}(p)$ such that the condition (3) holds, let $\lambda, \vartheta \in \mathbb{R}$ and $\gamma \in[0,1)$. If

$$
\operatorname{Re} \Omega_{\eta, p, q}^{n, m}(\lambda, \vartheta) f(z)>\mu(p, \lambda, \vartheta ; \gamma), z \in \mathbb{D},
$$

where

$$
\mu(p, \lambda, \vartheta ; \gamma):=\left\{\begin{array}{lll}
p(\lambda+\vartheta)-\frac{\gamma}{2(1-\gamma)}, & \text { if } & \gamma \in\left[0, \frac{1}{2}\right], \\
p(\lambda+\vartheta)-\frac{1-\gamma}{2 \gamma}, & \text { if } & \gamma \in\left[\frac{1}{2}, 1\right),
\end{array}\right.
$$

then

$$
\operatorname{Re}\left\{\left[\frac{\left(\mathfrak{S}_{\eta, p, q}^{n, m} f(z)\right)^{\prime}}{p z^{p-1}}\right]^{\lambda}\left[\frac{\mathfrak{S}_{\eta, p, q}^{n, m} f(z)}{z^{p}}\right]^{\vartheta}\right\}>\gamma, z \in \mathbb{D},
$$

that is $f \in A_{\eta, p, q}^{n, m}(\lambda, \vartheta ; \gamma)$. 
Proof. If we define the function $\mathrm{p}$ by

$$
\mathrm{p}(z)=\frac{1}{1-\gamma}\left(\left[\frac{\left(\mathfrak{S}_{\eta, p, q}^{n, m} f(z)\right)^{\prime}}{p z^{p-1}}\right]^{\lambda}\left[\frac{\mathfrak{S}_{\eta, p, q}^{n, m} f(z)}{z^{p}}\right]^{\vartheta}-\gamma\right), z \in \mathbb{D},
$$

from the assumption (3) we deduce that the function $p$ is analytic in $\mathbb{D}$, with $p(0)=1$, hence $p \in \mathcal{H}[1,1]$. From the definition relation (9) it is easy to check that

$$
\Omega_{\eta, p, q}^{n, m}(\lambda, \vartheta) f(z)=p(\lambda+\vartheta)+\frac{(1-\gamma) z \mathrm{p}^{\prime}(z)}{(1-\gamma) \mathrm{p}(z)+\gamma}, z \in \mathbb{D}
$$

Denoting

$$
\begin{aligned}
& \psi(r, s ; z):=p(\lambda+\vartheta)+\frac{(1-\gamma) s}{(1-\gamma) r+\gamma}, s \in \mathbb{C}, r \in \mathbb{C} \backslash\left\{-\frac{\gamma}{1-\gamma}\right\} \\
& \Omega:=\{w \in \mathbb{C}: \operatorname{Re} w>\mu(p, \lambda, \vartheta ; \gamma)\}
\end{aligned}
$$

the assumption (6) is equivalent to $\psi\left(\mathrm{p}(z), z \mathrm{p}^{\prime}(z) ; z\right)=\Omega_{\eta, p, q}^{n, m}(\lambda, \vartheta) f(z) \in \Omega$ for all $z \in \mathbb{D}$.

Also, for any $x, y \in \mathbb{R}$ with $y \leq-\left(1+x^{2}\right) / 2$, and $z \in \mathbb{D}$ a simple computation shows that

$$
\begin{aligned}
& \operatorname{Re} \psi(i x, y ; z)=p(\lambda+\vartheta)+\frac{\gamma(1-\gamma) y}{(1-\gamma)^{2} x^{2}+\gamma^{2}} \\
& \leq p(\lambda+\vartheta)-\frac{\gamma(1-\gamma)}{2} \frac{x^{2}+1}{(1-\gamma)^{2} x^{2}+\gamma^{2}}=: h(x) \\
& \leq \sup \{h(x): x \in \mathbb{R}\}=\left\{\begin{array}{lll}
\lim _{x \rightarrow+\infty} h(x), & \text { if } & \gamma \in\left[0, \frac{1}{2}\right], \\
h(0), & \text { if } & \gamma \in\left[\frac{1}{2}, 1\right)
\end{array}=\mu(p, \lambda, \vartheta ; \gamma),\right.
\end{aligned}
$$

where $\mu(p, \lambda, \vartheta ; \gamma)$ is given by (7). Therefore, $\psi(i x, y ; z) \notin \Omega$ if $x, y \in \mathbb{R}$ with $y \leq-\left(1+x^{2}\right) / 2$, and $z \in \mathbb{D}$. Using Lemma 2 we conclude that $\operatorname{Re} p(z)>0$ for all $z \in \mathbb{D}$, that is the conclusion (8) holds.

If we set $\lambda:=\alpha$ and $\vartheta:=1-\alpha$ in Theorem 2 we obtain the following special case:

Corollary 3. Let the function $f \in \mathbb{A}(p)$ such that the condition (3) holds, let $\alpha \in \mathbb{R}$ and $\gamma \in[0,1)$. If

$$
\operatorname{Re} \Omega_{\eta, p, q}^{n, m}(\alpha, 1-\alpha) f(z)>\widetilde{\mu}(p, \alpha, ; \gamma):=\mu(p, \alpha, 1-\alpha ; \gamma), z \in \mathbb{D},
$$

where $\mu(p, \lambda, \vartheta ; \gamma)$ is defined by $(7)$, then

$$
\operatorname{Re}\left\{\left[\frac{\left(\mathfrak{S}_{\eta, p, q}^{n, m} f(z)\right)^{\prime}}{p z^{p-1}}\right]^{\alpha}\left[\frac{\mathfrak{S}_{\eta, p, q}^{n, m} f(z)}{z^{p}}\right]^{1-\alpha}\right\}>\gamma, z \in \mathbb{D},
$$

that is $f \in A_{\eta, p, q}^{n, m}(\alpha, 1-\alpha ; \gamma)$.

Remark 3. The above corollary could be written as follows:

Let the function $f \in \mathbb{A}(p)$ such that the condition (3) holds, let $\alpha \in \mathbb{R}$ and $\gamma \in[0,1)$. If $f \in$ $M_{\eta, p, q}^{n, m}(\alpha ; \mu(p, \alpha, 1-\alpha ; \gamma))$ where $\mu(p, \lambda, \vartheta ; \gamma)$ is given by $(7)$, then $f \in A_{\eta, p, q}^{n, m}(\alpha, 1-\alpha ; \gamma)$.

If we set $\lambda=-1$ and $\vartheta=1$ in Theorems 1 and 2 we next get the following corollaries, respectively. 
Corollary 4. Let the function $f \in \mathbb{A}(p)$ such that the condition (3) holds, and let $N \geq 1$. Then

$$
\operatorname{Re} \Omega_{\eta, p, q}^{n, m}(-1,1) f(z)<\frac{N}{N+1}, z \in \mathbb{D},
$$

implies

$$
\left|\frac{\mathfrak{S}_{\eta, p, q}^{n, m} f(z)}{z\left(\mathfrak{S}_{\eta, p, q}^{n, m} f(z)\right)^{\prime}}-\frac{1}{p}\right|<\frac{N}{p}, z \in \mathbb{D},
$$

or equivalently

$$
\begin{aligned}
& \left|\frac{z\left(\mathfrak{S}_{\eta, p, q}^{n, m} f(z)\right)^{\prime}}{\mathfrak{S}_{\eta, p, q}^{n, m} f(z)}+\frac{p}{N^{2}-1}\right|>\frac{p N}{N^{2}-1}, z \in \mathbb{D}, \text { if } N>1, \\
& \operatorname{Re} \frac{z\left(\mathfrak{S}_{\eta, p, q}^{n, m} f(z)\right)^{\prime}}{\mathfrak{S}_{\eta, p, q}^{n, m} f(z)}>\frac{p}{2}, z \in \mathbb{D}, \text { if } \quad N=1 .
\end{aligned}
$$

Corollary 5. Let the function $f \in \mathbb{A}(p)$ such that (3) holds, and let $\gamma \in[0,1)$. If

$$
\operatorname{Re} \Omega_{\eta, p, q}^{n, m}(-1,1) f(z)>\mu(p,-1,1 ; \gamma), z \in \mathbb{D},
$$

where $\mu(p, \lambda, \vartheta ; \gamma)$ is defined by $(7)$, then

$$
\operatorname{Re} \frac{\mathfrak{S}_{\eta, p, q}^{n, m} f(z)}{z\left(\mathfrak{S}_{\eta, p, q}^{n, m} f(z)\right)^{\prime}}>\frac{\gamma}{p}, z \in \mathbb{D},
$$

or equivalently

$$
\begin{aligned}
& \left|\frac{z\left(\mathfrak{S}_{\eta, p, q}^{n, m} f(z)\right)^{\prime}}{\mathfrak{S}_{\eta, p, q}^{n, m} f(z)}-\frac{p}{2 \gamma}\right|<\frac{p}{2 \gamma}, z \in \mathbb{D}, \text { if } \gamma \in(0,1), \\
& \operatorname{Re} \frac{z\left(\mathfrak{S}_{\eta, p, q}^{n, m} f(z)\right)^{\prime}}{\mathfrak{S}_{\eta, p, q}^{n, m} f(z)}>0, z \in \mathbb{D}, \text { if } \quad \gamma=0 .
\end{aligned}
$$

\section{Conclusions}

The novelty of the above results consists in the fact that the new defined operators $\mathfrak{S}_{\eta, p, q}^{n, m}$ generalize and extend many previously studied operators by different authors.

This operator was used to define two new subclasses of functions, and we found sufficient conditions for a function $f \in \mathbb{A}(p)$ to belong to these classes by using classical results of the general theory of differential subordinations. These subclasses of multivalent functions could be connected with those mentioned in Remark 1 and extend the classes of Remark 2, while the investigation methods consisting of the two lemmas are more powerful than those used by the previous authors.

Moreover, for appropriate choices of the parameters, both of the above theorems give us simple sufficient conditions for a function $f \in \mathbb{A}(p)$ to belong to different subclasses of $A_{\eta, p, q}^{n, m}(\lambda, \vartheta ; \gamma)$.

Author Contributions: Conceptualization, E.E.A. and T.B.; methodology, E.E.A. and T.B.; investigation, E.E.A. and T.B.; resources, E.E.A. and T.B.; writing—original draft preparation, E.E.A. and T.B.; writing-review and editing, E.E.A. and T.B.; supervision, E.E.A. and T.B.; project administration, E.E.A. and T.B. The authors contributed equally to this work. All authors have read and agreed to the published version of the manuscript.

Funding: This research received no external funding.

Conflicts of Interest: The authors declare no conflict of interest. 


\section{References}

1. Jackson, F.H. On $q$-functions and a certain difference operator. Trans. R. Soc. Edin. 1909, 46, $253-281$. [CrossRef]

2. Jackson, F.H. On q-definite integrals. Quart. J. Pure Appl. Math. 1910, 41, 193-203.

3. Frasin, B.A.; Murugusundarmoorthy, G. A subordination results for a class of analytic functions defined by q-differential operator. Ann. Univ. Paedagog. Crac. Stud. Math. 2020, 19, 53-64. [CrossRef]

4. Aouf, M.K.; Mostafa, A.O.; El-Ashwah, R.M. Sandwich theorems for $p$-valent functions defined by a certain integral operator. Math. Comput. Model. 2011, 53, 1647-1653. [CrossRef]

5. Aouf, M.K.; Mostafa, A.O. On a subclass of $n-p$-valent prestarlike functions. Comput. Math. Appl. 2008, 55, 851-861. [CrossRef]

6. Kamali, M.; Orhan, H. On a subclass of certain starlike functions with negative coefficients. Bull. Korean Math. Soc. 2004, 41, 53-71. [CrossRef]

7. Orhan, H.; Kiziltunç, H. A generalization on subfamily of $p$-valent functions with negative coefficients. Appl. Math. Comput. 2004, 155, 521-530. [CrossRef]

8. Sălăgean, G.S. Subclasses of univalent functions. In Complex Analysis, Fifth Romanian-Finnish Seminar; Part 1 (Bucharest); Springer: Berlin/Heidelberg, Germany, 1983; pp. 362-372.

9. Al-Oboudi, F.M. On univalent functions defined by a generalized Sălăgean operator. Int. J. Math. Math. Sci. 2004, 27, 1429-1436. [CrossRef]

10. Ul-Haq, M.; Raza, M.; Arif, M.; Khan, Q.; Tang, H. q-Analogue of differential subordinations. Mathematics 2019, 7, 724. [CrossRef]

11. Srivastava, H.M. Integral Transforms and Operational Calculus; MDPI Publishers: Basel, Switzerland, 2019; ISBN 978-3-03921-618-5 (Pbk); ISBN 978-3-03921-619-2 (PDF).

12. Srivastava, H.M. Operators of Fractional Calculus and Their Applications; MDPI Publishers: Basel, Switzerland, 2019; p. viii, 125p; ISBN 978-3-03897-340-9 (Pbk); ISBN 978-3-03897-341-6 (PDF).

13. Srivastava, H.M. Operators of basic (or $q$-) calculus and fractional $q$-calculus and their applications in Geometric Function Theory of Complex Analysis. Iran. J. Sci. Technol. Trans. A Sci. 2020, 44, 327-344. [CrossRef]

14. Patil, D.A.; Thakare, N.K. On convex and extreme point of $p$-valent starlike and convex classes with application. Bull. Math. Soc. Sci. Math. Roumanie (N.S.) 1983, 27, 145-160.

15. Owa, S. On certain classes of $p$-valent functions with negative coefficients. Bull. Belg. Math. Soc. Simon Stevin 1985, 59, 385-402.

16. Irmak, H.; Bulboacă, T.; Tuneski, N. Some relations between certain classes consisting of $\alpha$-convex type and Bazilević type functions. Appl. Math. Lett. 2011, 24, 2010-2014. [CrossRef]

17. Miller S.S.; Mocanu, P.T. Differential Subordinations. Theory and Applications; Series of Monographs and Textbooks in Pure and Applied Mathematics; Marcel Dekker Inc.: New York, NY, USA, 2000; Volume 225.

Publisher's Note: MDPI stays neutral with regard to jurisdictional claims in published maps and institutional affiliations.

(C) 2020 by the authors. Licensee MDPI, Basel, Switzerland. This article is an open access article distributed under the terms and conditions of the Creative Commons Attribution (CC BY) license (http://creativecommons.org/licenses/by/4.0/). 